\title{
Revealing the onset of free convection in terrestrial planet atmospheres
}

\author{
Sebastian G. Els ${ }^{1}$ and Konstantinos Vogiatzis ${ }^{2}$ \\ ${ }^{1}$ AURA-NIO, Cerro Tololo Interamerican Observatory, Casilla 603, La Serena, Chile \\ email: sels@ctio.noao.edu \\ ${ }^{2}$ AURA-NIO, National Optical Astronomy Observatory, \\ 950 N. Cherry Ave., Tucson, AZ 85719, USA \\ email: voyages@noao.edu
}

\begin{abstract}
The site testing program for the Thirty-Meter-Telescope (TMT) is monitoring the seeing conditions above several mountains. Observations are presented which show that the ground layer seeing $(0 \mathrm{~m}-500 \mathrm{~m}$ above the ground) is influenced by the onset of free convection. It is shown that under radiation dominated conditions, indicating free convection of the atmosphere above the site, the occurence of the very best seeing conditions is supressed. A quantitative threshold for these conditions is given, allowing to estimate the shortterm future stability of the ground layer seeing.
\end{abstract}

Keywords. Atmospheric effects, convection, turbulence, Earth

\section{Introduction}

One of the most important parameters indicating the quality of a potential astronomical site is seeing. The standard instrument measuring the integrated seeing through the entire atmosphere above the telescope level is the Differential Image Motion Montior (DIMM, Tokovinin 2002). In recent years the Multi Aperture Scintillation Sensor (MASS, Tokovinin et al. 2003) has been developed allowing to measure $C_{N}^{2}(h)$ turbulence profiles on a routine basis. The site testing campaign for the TMT employs a combined MASSDIMM device on their site testing telescopes, mounted $z=7 \mathrm{~m}$ above the ground. The TMT site testing equipment also consists of a meteorological station measuring basic atmospheric parameters as temperature $T$, relative humidity, atmospheric pressure $P$, wind speed $v$ and direction at an elevation of $z=2 \mathrm{~m}$ (Skidmore et al. 2004). Additional instrumentation, in part installed temporarily, consists of all-sky cameras (Walker et al. 2006), SODAR (Travouillon 2006) and sonic anemometers (Skidmore et al. 2006).

\section{Seeing and convection of the first $500 \mathrm{~m}$}

The TMT MASS delivers the seeing as measured above $z=500 \mathrm{~m}$. From the simultaneously obtained MASS and DIMM seeing measurements $\left(s_{M}, s_{D}\right.$, respectively) it is possible to compute the seeing within the first $500 \mathrm{~m}$ above the ground by $s_{G L}=$ $\left(s_{D}^{5 / 3}-s_{M}^{5 / 3}\right)^{3 / 5}$, which we will refer to as groundlayer GL. As pointed out by Els \& Vogiatzis (2006) the energy flux emerging from the ground prevents the groundlayer seeing to reach its very best values. Here we make use of the ground heat flux, as measured a few $\mathrm{cm}$ within the ground and the net radiation flux measured $2 \mathrm{~m}$ above the surface. The difference $H$ of these two values gives the energy deposited into the air just above the ground. In the following, data are shown which have been collected between November 2004 and August 2006 at Cerro Armazones (3064 m ASL) in northern Chile. In Fig. 1 


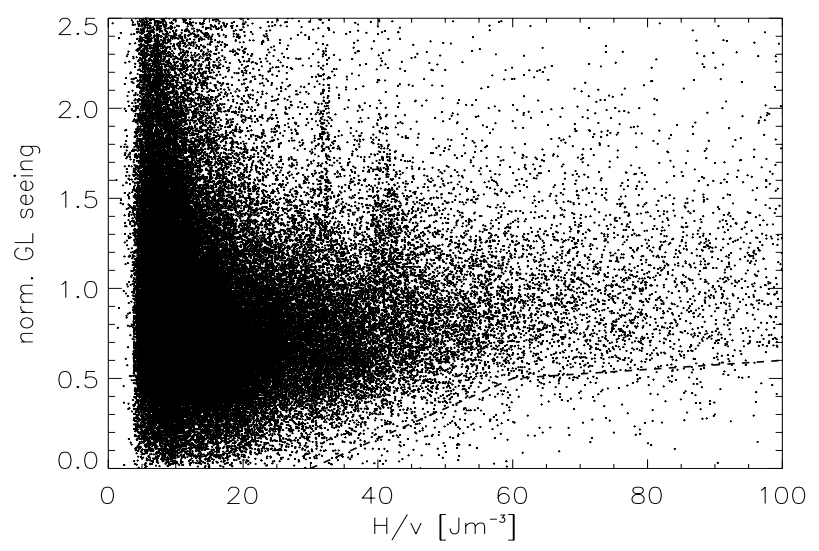

Figure 1. Normalized groundlayer seeing $\left(s_{G L} / \bar{s}_{G L}\right)$ versus $H / v(z=2 \mathrm{~m})$. The occurence of the best seeing values is suppressed beyond $H / v \gtrsim 30 \mathrm{Jm}^{-3}$; the dashed line approximately outlines this trend.

the dependence of $s_{G L}$ (normalized by the average $\bar{s}_{G L}$ ) on the quotient $H / v(z=2 \mathrm{~m}$ ) is shown. In the region $H / v(z=2 \mathrm{~m}) \gtrsim 30 \mathrm{Jm}^{-3}$ the very best values $\left(s_{G L} \lesssim 0.3 \cdot \bar{s}_{G L}\right)$ are not reached. The seeing in this region is just about (and slightly better) than the overall average value $\bar{s}_{G L}$. In order to investigate whether this effect is due to free convection the Monin-Obukhov length $L_{\star}=-\frac{u^{3} T \rho c_{p}}{\kappa g Q_{H}}$ is used (Monin \& Obukhov 1954). Where $Q_{H}=H /\left(c_{p} \rho\right)$ is the sensible heatflux from the ground, $\rho$ the atmospheric density and $c_{p}$ the heat capacity of air. $u$ is the friction velocity $u=v(z) / \ln \left(z / z_{0}\right)$. With $v$ measured at $z=2 \mathrm{~m}$ and an assumed roughness of $z_{0}=0.005 \mathrm{~m}$, corresponding to surface features of about $5 \mathrm{~cm}$ in size. $-L_{\star}$ gives the height above ground at which the turbulent energy production rate of the dynamic interaction of the air with the ground is equal to turbulent energy production rate due to buoyancy. In Fig. 2 the groundlayer seeing $s_{G L}$ versus $L_{\star}$ is shown for data for which $H / v(z=2 \mathrm{~m}) \gtrsim 30 \mathrm{Jm}^{-3}$. All of these data fall at $-L_{\star}<8 \mathrm{~m}$, and mostly $-L_{\star}<1 \mathrm{~m}$. Therefore the condition for free convection, $z / L_{\star}<-1$, is fulfilled already very close to the ground.

\section{Summary and conclusion}

It was shown that the occurence of optimal (much better than average) GL seeing is supressed under atmospheric conditions in agreement with free convection at the telescope level. The extension of the dynamic layer of the atmosphere above the telescope is thus necessary to obtain the best possible seeing conditions. Future measurements of the $3 \mathrm{D}$ wind speeds and temperature profiles at several elevations using a $30 \mathrm{~m}$ tower, installed in the vicinity of the telescope, will allow to improve the measurement of $L_{\star}$. The easy to measure quotient of $H / v$ might be used as an indicator for the stability of the atmospheric conditions and can therefore be employed to estimate the forthcoming shortterm seeing conditions above a site. This work did not take any effects of potential structures surrounding the telescope (i.e., dome, trees) into account and to make use of that parameter on other astronomical sites requires a proper calibration for such effects. Whether a modification of the surroundings of the telescope (i.e., painting of the surface) is sufficient to decrease the occurance of free convection remains to be seen during future investigations. 


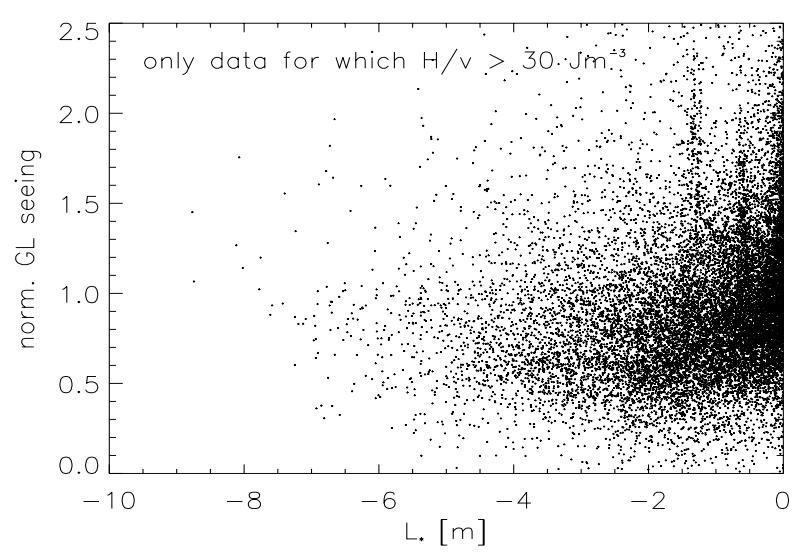

Figure 2. Normalized groundlayer seeing versus the Monin-Obukhov length $L_{\star}$ only for data which fall beyond $H / v(z=2 \mathrm{~m}) \gtrsim 30 \mathrm{Jm}^{-3}$ in Fig. 1 . All data fall at $-L_{\star} \lesssim 8 \mathrm{~m}$ and most of them even $-L_{\star} \lesssim 3 \mathrm{~m}$, indicating that the conditions for an unstable or free convective atmosphere, $z / L<-1$, are reached very close to the ground.

\section{Acknowledgements}

We acknowledge the effort of the entire TMT site testing team making this work possible. The authors gratefully acknowledge the support of the TMT partner institutions. They are the Association of Canadian Universities for Research in Astronomy (ACURA), the Association of Universities for Research in Astronomy (AURA), the California Institute of Technology and the University of California. This work was supported, as well, by the Canada Foundation for Innovation, the Gordon and Betty Moore Foundation, the National Optical Astronomy Observatory, which is operated by AURA under cooperative agreement with the National Science Foundation, the Ontario Ministry of Research and Innovation, and the National Research Council of Canada.

\section{References}

Els, S.G. \& Vogiatzis, K. 2006, SPIE 6267, 67

Monin, A.M. \& Obukhov, A.M. 1954, Geofiz. Inst. Akad. Nauk. SSSR 24(151), 163

Skidmore, W., Schoeck, M., Tokovinin, A., et al. 2004, SPIE 5489, 154

Skidmore, W., Travouillon, T. \& Riddle, R. 2006, SPIE 6267, 64

Tokovinin, A. 2002, PSAP 114, 1156

Tokovinin, A., Kornilov, V., Shatsky, N. \& Vozyakova, O. 2003, MNRAS 434, 891

Travouillon, T. 2006, SPIE 6267, 65

Walker, D.E., Schwarz, H. \& Bustos, E. 2006, SPIE 6267, 87 